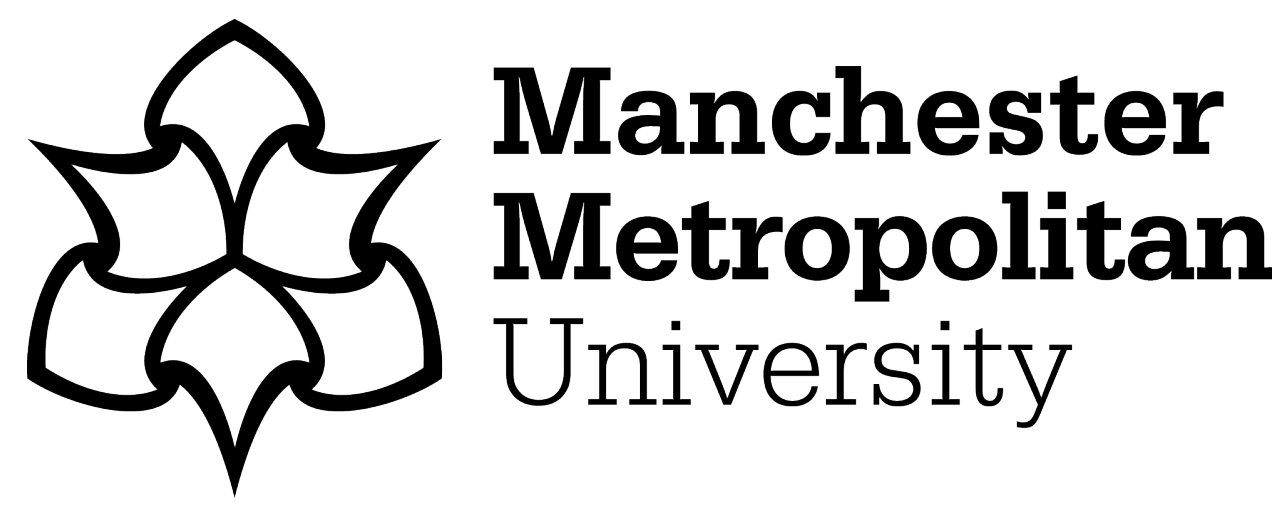

Curry, Collette, O'Shea, Jim ORCID logoORCID: https://orcid.org/00000001-5645-2370 and Crockett, Keeley ORCID logoORCID: https://orcid.org/0000-0003-1941-6201 (2020) Do You Remember Me? Betty the Conversational Agent. In: Proceedings of the 40th SGAI International Conference on Artificial Intelligence, Al 2020, 15 December 2020 - 17 December 2020, Cambridge, UK.

Downloaded from: https://e-space.mmu.ac.uk/627062/

Publisher: Springer

DOI: https://doi.org/10.1007/978-3-030-63799-6_32

Please cite the published version 


\title{
Do You Remember Me? Betty the Conversational Agent
}

\author{
Collette Curry ${ }^{(凶)}$ [0000-0001-9692-0212], James D O’Shea, Keeley Crockett ${ }^{[0000-0003-1941-6201]}$ \\ Department of Computing \& Maths, Manchester Metropolitan University, UK \\ drccurryebtinternet.com \\ j.d.oshea@mmu.ac.uk \\ k.crockett@mmu.ac.uk
}

\begin{abstract}
In this paper we introduce a conversational agent reminiscence companion system called Betty. Betty increases subjective wellbeing (SWB) by engaging with people and by collecting information during one-to-one conversations, then using this data in further personalised conversations with each individual. Results have shown a positive effect on SWB, and improvement in normal age-associated memory loss (NM) was evident through increased everyday interaction with Betty.
\end{abstract}

Keywords: Conversational Agent, Subjective Wellbeing, Reminiscence

\section{Introduction}

In this paper we introduce the development of Betty, a reminiscence themed conversational agent. Throughout development of Betty, a series of experiments were conducted, and results suggested an increase in SWB and improvement in NM in participants aged 65 years and older.

\subsection{Ageing population growth}

In 2017, $18.2 \%$ of the United Kingdom (UK) population were aged 65 years or older. More than a quarter of UK residents will be 65 years or over within the next 50 years [1]. As the population ages, normal ageing memory impairment problems will become more acute [2]. Improving memory impairment reduces distress for individuals and enhances their wellbeing and independence [3,4].

The quality of life and moods in old age can be improved by increasing Subjective Wellbeing [5]. Moods are subjective states of mind that are typically described and quantified using self-reporting measures [6].

\subsection{Conversational Agent Framework}

A suitable methodology was followed to create Betty. The first factor considered was 'What is such a system going to offer?'. Betty would provide information, answer questions, provide companionship and discuss different reminiscence domain themed topics. The direction of the interaction would be dual, so that the user and also Betty 
could introduce topics into the conversation. Betty would maintain the conversation through an Eliza layer and new content would be added to the personal ontology knowledge base saved for each user. Betty would allow some 'small-talk' and would steer the conversation when required. The handling of unknown or unexpected user input needed to be addressed and handling such content was built into the system. This was to ensure that an appropriate response was given at all times. This also allowed errors to be captured and to be handled by Betty and conversation continue uninterrupted.

Each participant would log in to access their personal ontology, which would build up over time, and to access a 'Learn' mode which would be set to run for either a set period of time during experimental studies, or until the user ended the current conversation. A modular approach was adopted in terms of themed reminiscence ontology inclusion. Content for the initial themed reminiscence ontology was gathered during group discussions, use of a questionnaire and also from the experimental conversational logs.

\section{Related Work}

There are many themed chatbots and conversational agents but none within the reminiscence domain. Systems are generally constructed by teams of people working on scripted replies and responses to input. There is a specific goal in mind when the system is produced. This could be to inform on a product or service or to give information about a set domain.

\section{Developing Betty}

Betty was developed to engage older people in conversation using reminiscence. Intended use of this system was to act as a personal companion that could allow conversation at any time of the day or night. Betty was non-judgemental, was never tired, and was always available to talk to. Conversations could be carried out and freely revisited at any time [7].

\subsection{Components of the system}

Components and functions of the system included the following.

\section{Eliza Layer.}

This layer kept the conversation on track and offered general conversation content to promote discussion. Conversation was sustained and continued throughout each session. Content that was recognised was redirected to the 'rule matcher', whilst unknown content was collected, added to the system and increased each personal ontology. If the system was in 'learn' mode, the system learned new content and reused the learnt content in further conversation with each individual. In general mode, the 
Eliza layer remembered the previous 25 iterations only, so giving an impression of recalling what had been said recently.

\section{Rule Matcher.}

User input through conversation was checked by the rule matcher which accepted the input and returned an appropriate response. The rules consisted of different topics and concepts. There were more than 20,000 rules mapped to the Reminiscence Ontology and WordNet.

\section{Reminiscence Ontology.}

This themed domain promoted recall and continued reminiscence engagement during conversation. Some subjects were commonly referred to, such as, 'Sixties cars', Schooldays', 'Family', 'Weather', and 'Sixties Music'. The reminiscence ontology is the knowledgebase for the themed conversation and also for subsequent threads. It allowed for shorter lines of programmed code. This was achieved by referencing the contents as a dictionary of terms back within the script. Data was contained as a hierarchy of tagged classes which were mapped to WordNet. The system automatically acted upon these tagged classes associations. Reminiscence domain known classes were split into attributes and roles. An ontology search algorithm processed user input, searching for named classes that were a match to detected keywords, it then further refined user input by identifying related terms. A number of subset assertions restricted the number of possible meanings and therefore resulted in more accurate and appropriate matched responses. Starting conversation is sometimes difficult for people, so having a supply of themed subjects to talk about helped participants. Scripted historical stories were incorporated to promote engagement and elicit more reminiscence data. These included events from the war years; the fifties and sixties; music and bands in the past; cars; sports, and famous people from the past.

\section{WordNet.}

A widely used resource in natural language processing. Betty was mapped to WordNet by identifying concepts then converting them to readable text files, sorted alphabetically with dates numbered. Within the companion system, WordNet was invoked by calling a keyword. For example, the conversation input could read "I remember the coalman bringing the coal", system reacted to the keyword 'coalman'. Found the definition and returned this internally to the system that then interrogated the reminiscence ontology and found more information about 'coalman'. The system replied, 'Did the coalman put the coal in the coal hole?', or 'Did the coalman bring the coal in a big sack?'.

\section{Conversation Manager.}

Accepts conversational input and passes it to the 'Eliza' layer or direct to the 'rule matcher'. When the conversation was a return conversation, Betty invoked past conversation, if two of these were refused by the person having the conversation, then the conversation manager returned the conversation to the Eliza layer and attempted to continue the conversation. 


\section{Spell checking.}

This attempted to correctly understand what had been input, even when spelled incorrectly. The input was checked, and the correct response then returned. The Eliza layer was invoked by unknown input to attempt to elicit further information which could then be interpreted, and a correct reply given. A glossary of terms from the past was included within the spell checker.

\section{Short-term memory.}

Utilised the Eliza layer and allowed limited interactive unscripted conversation. The input was saved as a re-writable file that was progressively overwritten as new input was received.

\section{Long-term memory and the personal ontology.}

Some content was shared with others via the reminiscence ontology which increased through user conversations. An example would be a conversation about 'cleaning the step with a donkey stone'. Imagine that 'donkey stone' was unknown to Betty, so when added to the reminiscence ontology, it and its definition would be available to all people. Other conversation was stored as a personal ontology and was not available to anyone else.

\subsection{The system in operation}

Betty incorporated some standard components of conversational agents; novel features were also included. A generic diagram of the system in operation is illustrated in Figure 1.

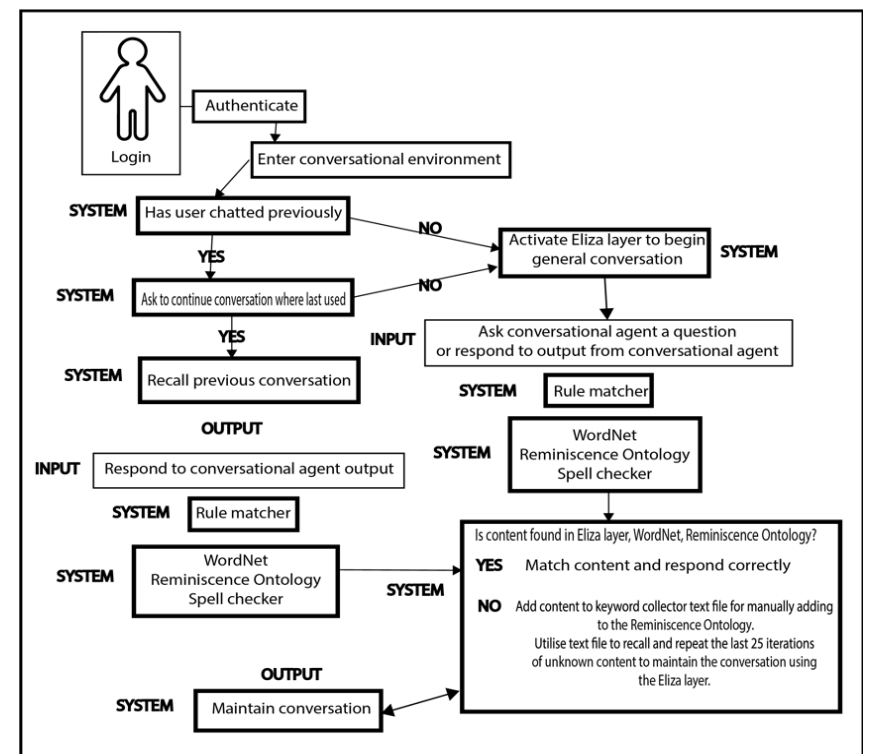

Fig. 1. Generic diagram of how the system worked. 


\section{Conclusions}

There was strong evidence to suggest that a CA used as a conduit for reminiscence is successful if used to improve NM and to increase SWB in participants. Application of the ontology of reminiscence has informed the knowledge of Betty and provided a way of including reminiscence within conversation. Further modular ontologies can be compiled and added to expand the conversational topics and therefore allow more conversational themes to be explored by the participants conversing with Betty. The development of modular ontologies using new methods, combined with a novel scripting mechanism has shortened the time spent scripting Betty. The benefits of the use of a CA in the way described might include improved user memory, lack of isolation and new methods of social interaction in older people [7].

\section{References}

1. S. Coates,: Overview of the UK population: November 2018. Office for National Statistics, (2018).

2. A. Morse,: Improving Dementia Services in England: An Interim Report, National Audit Office, (2010).

3. M. Dorin,: Online education of older adults and its relation to life satisfaction. In: Educational Gerontology, vol. 33, pp. 127-143, (2007).

4. J. H. Wagner, K. Hassanein and M. Head,: Computer use by older adults: A multidisciplinary review. In: Computers in Human Behavior, vol. 26, pp. 870-882, (2010). doi:

5. L. K. George,: Still Happy After All These Years: Research Frontiers on Subjective Wellbeing in Later Life. In: Journals of Gerontology Series B-Psychological Sciences and Social Sciences, vol. 65, pp. 331-339, (2010).

6. L. J. E. Brown and A. J. Astell,: Assessing mood in older adults: a conceptual review of methods and approaches. In: International Psychogeriatrics, vol. 24, no. 8, pp. 1197-1206, (2012).

7. C. Curry,: A framework for developing a CA to improve age associated memory loss and increase subjective wellbeing, $\mathrm{PhD}$ Thesis, Manchester Metropolitan University, (2018). 